Apidologie, 1976, 7 (3), 237-242.

\title{
SUR DES SUBSTANCES QUI PROVOQUENT L'ÉTIRAgE DE LA CIRE
}

\author{
Über Substanzen, die das Ausbauen der Waben bewirken \\ Rémy CHAUVIN \\ Station d'Éthologie \\ Mittainville 78120 Rambouillet
}

SUMMARY

ABOUT SOME SUBSTANCES INDUCING WAX STRETCHING

In alcohol extracts of pollen and in water extract of wax there are some substances that stimulate wax stretching by caged bees.

\section{RÉSUMÉ}

Il existe dans l'extrait alcoolique de pollen et dans les eaux d'extraction de la cire des substances qui excitent l'étirage de la cire parmi les abeilles en cagettes.

\section{INTRODUCTION}

L'étirage de la cire chez les abeilles nourries en cagettes à l'étuve à $33^{\circ}$ a été étudiée par un grand nombre d'auteurs (Freudenstein, Darchen, Frichot-Riera). Mais on ne sait que peu de choses du déterminisme physiologique de l'étirage, quoique l'existence d'une hormone de construction ait été signalée (Chauvin, Darchen et Pain, 1961). J'ai pu découvrir, un peu par hasard, l'action sur l'étirage d'une série de substances, distribuées en nourrissement aux abeilles. 


\section{MATÉRIEL ET MÉTHODES}

Les ouvrières employées étaient nées à l'étuve à partir de cadres de couvain prélevés dans les ruches, de mai à août 1974. Elles étaient réparties dans des cagettes de Pain, où elles ne recevaient que du candi; un carré de cire gaufrée de $4 \times 4 \mathrm{~cm}$ était fixé au haut de la cagette. Un abreuvoir de 3 ec contenait du sirop de sucre (conc. $500 \mathrm{~g}$ par litre), le sirop étant additionné, suivant les expériences, de diverses substances.

L'étirage était mesuré en détachant au bout de cinq jours à l'aide d'une lame de rasoir toute la cire étirée. Évidemment, la méthode n'est pas susceptible d'une grande précision. Mais comme on le verra, les différences observées sont si grandes qu'on peut s'en contenter.

\section{RÉSULTATS}

J'ai d'abord essayé de déterminer quel était le nombre optimum d'abeilles qu'il convenait d'introduire dans les cagettes pour obtenir le meilleur résultat (Tableau 1).

TAB. 1. -- Quantité de cire étirée en fonction du nombre d'abeilles TAB. 1. - Menge des ausgebauten Wachses abhängig von der Zahl der Bienen

\begin{tabular}{|c|c|c|}
\hline $\begin{array}{l}\text { Début expérience } \\
\text { Versuchsbeginn }\end{array}$ & $\begin{array}{c}\text { Nombre d'abeilles } \\
\text { en cagettes } \\
\text { Anzahl gekäfigter Bienen }\end{array}$ & $\begin{array}{l}\text { Mg. de cire étirée } \\
\text { mg ausgebautes Wachs }\end{array}$ \\
\hline 14 juin & $\begin{array}{l}100 \\
200\end{array}$ & $\begin{array}{l}230 \\
562\end{array}$ \\
\hline 6 juillet & $\begin{array}{l}100 \\
200\end{array}$ & $\begin{array}{l}198 \\
274\end{array}$ \\
\hline 14 juillet & $\begin{array}{r}100 \\
200 \\
400 \\
50\end{array}$ & $\begin{array}{r}0(?) \\
527 \\
425 \\
63\end{array}$ \\
\hline 24 juillet & $\begin{array}{l}100 \\
200 \\
300 \\
400\end{array}$ & $\begin{array}{l}191 \\
485 \\
220 \\
179\end{array}$ \\
\hline 10 août & $\begin{array}{l}100 \\
200 \\
300 \\
360\end{array}$ & $\begin{array}{l}178 \\
462 \\
258 \\
255\end{array}$ \\
\hline 16 août & $\begin{array}{l}200 \\
300 \\
400\end{array}$ & $\begin{array}{r}478 \\
176 \\
77\end{array}$ \\
\hline 18 août & $\begin{array}{l}100 \\
200\end{array}$ & $\begin{array}{l}225 \\
415\end{array}$ \\
\hline
\end{tabular}


Si l'on ramène la quantité de cire étirée au chiffre par individu, en négligeant le chiffre du 14 juillet pour 100 abeilles qui est sûrement anormal, on obtient les résultats exposés dans le tableau 2.

Tав. 2. - Quantité de cire étirée par abeille selon le nombre d'abeilles encagées

TAв. 2. - Ausgebaute Wachsmenge je Biene nach Anzahl der gekäfigten Bienen

\begin{tabular}{c|c}
$\begin{array}{c}\text { Nombre d'abeilles } \\
\text { Anzahl Bienen }\end{array}$ & $\begin{array}{c}\text { Mg. de cire étirée par abeille } \\
\text { mg ausgebautes Wachs je Biene }\end{array}$ \\
\hline 100 & 2,07 \\
200 & 2,2 \\
300 & 0,72 \\
400 & 0,56 \\
\hline
\end{tabular}

Il est donc évident qu'à partir de 300 abeilles se produisent des phénomènes d'encombrement (qui n'entraînent cependant aucune mortalité pas plus que pour 400 , tout au moins au bout de 5 jours). D'autre part, le groupement de 200 abeilles n'amène pas un étirage bien supérieur au groupe de 100. C'est pourquoi je m'en suis tenu au groupe de 100 abeilles par la suite.

\section{ESSAIS DE SUBSTANCES ACTIVES}

J'ai constaté au début de 1974, et par hasard, que les extraits alcooliques de pollen avaient une influence sur l'étirage (et aussi, me semble-t-il, sur la fécondité de la reine et la santé générale de la ruche, mais je n’ai pas assez de résultats ici pour me montrer affirmatif). Ces extraits sont préparés en faisant bouillir à reflux un mélange de pollens variés et séchés (surtout arbres fruitiers) récoltés par les abeilles dans les trappes à pollen; $500 \mathrm{~g}$ de pollen sont ainsi mis à bouillir à reflux dans deux litres d'alcool à $95^{\circ}$ pendant un quart d'heure. On obtient une solution rouge orangée dont une partie précipite rapidement par refroidissement. Pour les administrer aux abeilles on introduit 2,5 cc de cette solution dans 100 cc de sirop; il se produit immédiatement une émulsion stable. Les témoins reçoivent évidemment le même sirop plus $2,5 \% \mathrm{~d}$ 'alcool.

J'ai essayé par comparaison l'extrait aqueux de pollen obtenu en faisant macérer $500 \mathrm{~g}$ des mêmes pollens dans deux litres d'eau pendant la nuit; après filtration on obtient une solution brunâtre riche en protides. Je l'ai employée soit pure, soit à $2,5 \%$ dans le sirop de sucre. 
Une solution de propolis a été préparée également par décoction de propolis brute dans l'alcool bouillant jusqu'à saturation de l'alcool. Des matières cireuses précipitent dès le refroidissement; on les sépare par filtration. La solution est employée également à $2,5 \%$.

L'eau noire qui provient de l'extraction des vieilles cires par l'eau bouillante a été préparée en faisant bouillir une livre de vieux rayons très noirs dans deux litres d'eau pendant vingt minutes et en filtrant à chaud sous pression. La solution a été utilisée à raison de 6 cc pour $100 \mathrm{cc}$ de sirop (concentration optimum déterminée par tâtonnement).

Le tableau 3 exprime les résultats, tous obtenus avec 100 abeilles par cagette.

TAB.L 3. - Influence des extraits aqueux et alcoolique de pollen, de l'eau de cire et de la propolis sur l'étirage de la cire $\mathrm{Mg}$ de cire étirée par 100 abeilles

TAB. 3. - Einfluss der wässrigen und alkoholischen Extrakte von Pollen und Propolis sowie des Waschwassers von Waben auf das Ausbauen der Waben mg ausgebautes Wachs je 100 Bienen

\begin{tabular}{|c|c|c|c|c|c|c|c|c|}
\hline & \multicolumn{8}{|c|}{$\begin{array}{l}\text { Expériences } \mathbf{n}^{\circ} \\
\text { Nummern der Versuehe }\end{array}$} \\
\hline & 1 & 2 & 3 & 4 & 5 & 6 & 7 & 8 \\
\hline $\begin{array}{l}\text { Témoin } \\
\text { Kontrolle }\end{array}$ & 230 & 198 & 191 & 178 & 255 & 121 & 183 & 85 \\
\hline $\begin{array}{l}\text { Extrait aqueux de pollen } \ldots \ldots \ldots \ldots \\
\text { Wässriger Pollenextrakt }\end{array}$ & & & 0 & 312 & 186 & & 542 & 0 \\
\hline $\begin{array}{l}\text { Extrait alcoolique de pollen } \ldots \ldots \ldots \\
\text { Alkoholischer Pollenextrakt }\end{array}$ & 528 & 330 & & & 411 & & 505 & 309 \\
\hline $\begin{array}{l}\text { Extrait aqueux de cire...... } \\
\text { Wässriger Wachsextrakt }\end{array}$ & & & & & 470 & 200 & & \\
\hline $\begin{array}{l}\text { Propolis } \\
\text { Kittharz }\end{array}$ & & & 275 & 286 & $\begin{array}{l}210 \\
440\end{array}$ & 45 & & \\
\hline
\end{tabular}

Du point de vue de la signification statistique de ces résultats, on a employé le test de Mann et Whitney, non paramétrique. Pour l'extrait alcoolique de pollen, $U=40$; les extraits alcooliques donnent donc un étirage de la cire très significatif. Pour l'eau de cire, $U=22$, le résultat est marginalement significa- 
tif, mais d'autres expériences ont été faites depuis qui vont dans le même sens et rendent le test significatif. Pour la propolis en fin, le résultat n'est pas significativement différent du témoin.

En résumé, l'extrait alcoolique de pollen et l'eau de cire contiennent une ou plusieurs substances qui à faible dose excitent fortement l'étirage de la cire chez les jeunes abeilles, par un mécanisme dont nous ne savons rien.

Du point de vue chimique, l'extrait alcoolique de pollen d'une part et l'extrait aqueux des cires noircies contiennent évidemment une foule de substances mal définies ou non encore étudiées. On trouve dans l'extrait alcoolique des sucres, des glucides, des stérols par exemple; dans l'eau de cire que j'ai étudiée jadis (Chauvin, 1962), toutes sortes de composés azotés et phosphorés tout à fait inconnus, et dont on ignore même la provenance (venant de l'abeille elle-même ou des pollens?). Tout ce que je puis dire, c'est que si l'on précipite l'extrait alcoolique de pollen par l'éther, l'activité passe dans le précipité. Enfin ce précipité aisément soluble dans l'eau, de même que l'eau noire ou l'extrait aqueux de pollen, provoque à forte concentration chez les abeilles un arrêt total de l'étirage accompagné d'un dégorgement de substances rougeâtres et cristallines (sucrées ?) sur les parois de la cage. Une certaine mortalité s'ensuit mais l'appétit pour le sirop n'est pas affecté, les abeilles continuent à absorber avec avidité ce sirop qui perturbe pourtant leur organisme. Les phénomènes pathologiques sont surtout marqués avec l'extrait aqueux pur de pollen. Les dégorgements sont si abondants que les abeilles peuvent s'engluer dedans.

Ces substances ne provoquent aucun développement des ovaires. On aurait pu s'y attendre pourtant, puisque les trois substances extrait aqueux de pollen, extrait alcoolique de pollen et extrait de cires vieillies contiennent de l'azote.

Il est intéressant de souligner que ces substances agissent à très faible concentration, quoique je n'aie pas cherché la concentration limite.

Reçu pour publication en avril 1976.

Eingegangen im April 1976.

\section{ZUSAMMENFASSUNG}

Bienen, in Gruppen zu 100 im Brutschrank gehalten, können innerhalb von fünf Tagen eine Mittelwand messbar ausbauen. Man hat sich dieses Versuchs bedient, um den Einfluss verschiedener, dem Futtersyrup zugesetzter Substanzen auf das Aushauen festzustellen. Es zeigte sich, dass Alkoholextrakt von Pollen und das Waschwasser alter Waben, in Dosen zu 2,5\% gegeben, das Ausziehen der Mittelwände beträchtlich beschleunigen. Man weiss nichts über die Natur dieser aktiven Stoffe; keinesfalls aber scheinen sie die Ovarentwicklung zu beeinflussen. 


\section{RÉFÉRENCES BIBLIOGRAPHIQUES}

Chauvin R., 1962. - Sur le noircissement des vieilles cires. Ann. Abeille, 5, 59-63.

Chauvin R., Darchen R., Pain J., 1961. - Sur l'existence d'une hormone de construction chez les abeilles. C. R. Acad. Sci., 223, 1135-1136.

Darchen R., 1959. - Les techniques de construction chez Apis mellifica. Ann. Sci. nat. Zool., $12^{\mathrm{e}}$ sér., 1, 113-209 (Thèse Sci. nat. Paris, 1957, $\mathrm{n}^{0} 787$ ).

Freudenstein K., 1937. - Die Wachserzeugung und der Wabenbau im Bienenvolk. Disch. Imkerführer 11, 154-156.

Freudenstein H., 1961. - Entwicklung und Leistung der Wachsdrüsen der Honigbienen. Biol. Zentralbl., 80, 479-4.92.

Frichot-Ricra M., 1961. - Les tests de Darchen sur la construction de la cire. Essai de quantification. C. R. Acad. Sci., 263, 334-336. 